\title{
VERNICIA FORDII y AleuRITES MOLUCCANus (Euphorbiaceae) en la Argentina. Naturalización y etNobotánica
}

\author{
HÉCTOR A. KELLER ${ }^{1}$, PABLO C. STAMPELLA², GUSTAVO DELUCCHI ${ }^{3}$ y JULIO A. \\ HURRELL ${ }^{4}$
}

\begin{abstract}
Summary: Vernicia fordii and Aleurites moluccanus (Euphorbiaceae) in Argentina. Naturalization and ethnobotany. This paper constitutes the first record of two Euphorbiaceae species naturalized in Argentina: Vernicia fordii (Hemsl.) Airy-Shaw (Misiones) and Aleurites moluccanus (L.) Willd (Misiones and Corrientes). Synonymy, descriptions, distribution and habitat, phenology, uses, local importance, ethnobotany, naturalization status, and reference materials are included.
\end{abstract}

Key words: Vernicia fordii, Aleurites moluccanus, oleaginous plants, naturalization, Argentinean Flora, Misiones, Corrientes.

\begin{abstract}
Resumen: Este trabajo constituye el primer registro de dos especies de Euphorbiaceae como naturalizadas en la Argentina: Vernicia fordii (Hemsl.) Airy-Shaw (Misiones) y Aleurites moluccanus (L.) Willd. (Misiones y Corrientes). Se incluyen sinonimias, descripciones, distribución y hábitat, fenología, utilidades, importancia local, datos etnobotánicos, estado de naturalización y material de referencia.
\end{abstract}

Palabras clave: Vernicia fordii, Aleurites moluccanus, plantas oleaginosas, naturalización, Flora Argentina, Misiones, Corrientes.

\section{INTRODUCCIÓN}

Euphorbiaceae, con 322 géneros y 8910 spp. (Govaerts et al., 2000), presenta amplia distribución mundial en zonas cálidas y templadas, e incluye especies de gran importancia económica; entre otras,

\footnotetext{
${ }^{1}$ Facultad de Ciencias Forestales, Universidad Nacional de Misiones - Instituto de Botánica del Nordeste, Casilla de Correo 209, 3400-Corrientes, Argentina. E-mail: hakeller2000@yahoo.com.ar

${ }^{2}$ Laboratorio de Etnobotánica y Botánica Aplicada (LEBA). Facultad de Ciencias Naturales y Museo, Universidad Nacional de La Plata, Calle 64 nro. 3, 1900-La Plata, Argentina. Becario UNLP. E-mail: pstampella@yahoo. com

${ }^{3}$ Facultad de Ciencias Naturales y Museo, Universidad Nacional de La Plata, Paseo del Bosque s.nro., 1900-La Plata, Argentina. E-mail: delucchi@fcnym.unlp.edu.ar ${ }^{4}$ Laboratorio de Etnobotánica y Botánica Aplicada (LEBA). Facultad de Ciencias Naturales y Museo, Universidad Nacional de La Plata, Calle 64 nro. 3, 1900-La Plata, Argentina. Investigador CONICET. E-mail: juliohurrell@ gmail.com
}

Manihot esculenta Crantz, y Hevea brasiliensis (Willd. ex A. Juss.) Müll. Arg. En la Argentina, se halla representada por 27 géneros y unas 214 especies (IBODA, 2011).

En este trabajo se presentan dos especies correspondientes a dos géneros que se citan por primera vez como naturalizadas para nuestro país: Vernicia fordii y Aleurites moluccanus. Ambas fueron introducidas en las primeras décadas del siglo XX por sus semillas oleaginosas, de las que se obtiene "aceite de tung", y como ornamentales. Se incluyen las descripciones, sinonimias, iconografía, distribución y hábitat, fenología, datos referidos a su etnobotánica y naturalización, y materiales de referencia.

Vernicia Lour. y Aleurites J.R. Forst. \& G. Forst. son dos géneros afines incluidos en las subfamilias Crotonoideae o Euphorbioideae según los autores (Stuppy et al. 1999; Welzen \& Stuppy, 1999; Radcliffe-Smith, 2001; Wurdack et al., 2005; Tokuoka, 2007; Thakur \& Patil, 2011; Secco et al., 2012). Vernicia comprende 3 especies originarias del Sudeste asiático, desde Myanmar hasta Indonesia, 
China y Japón; Aleurites presenta 2 especies: una endémica de Hawai, la otra de amplia distribución en las zonas cálidas de Asia y Oceanía (Stuppy et al. 1999; Govaerts et al., 2000; Li \& Gilbert, 2008; Welzen \& Chayamarit, 2012).

\section{Materiales y Métodos}

Esta contribución se basa en diversos viajes de estudio realizados en Misiones y Corrientes. Se consultaron bibliotecas y herbarios, y el material que documenta el trabajo realizado fue depositado en el Herbario CTES (Instituto de Botánica del Nordeste, Corrientes).

Se realizaron entrevistas etnobotánicas abiertas (Albuquerque \& Lucena, 2004), a los pobladores locales de las zonas donde fueron halladas las especies tratadas, con el fin de obtener información sobre su proceso de naturalización.

\section{Resultados}

1. Vernicia fordii (Hemsl.) Airy Shaw, Kew Bull. 20: 394, 1966. Fig. 1, A-H.

Aleurites fordii Hemsl., Hooker's Icon. Pl. 29: tab. 2801, 2802, 1906.

Iconografia: Hemsley, loc. cit.; Li \& Gilbert, 2008: fig. 320, 5-6.

Árboles hasta de $10 \mathrm{~m}$ alt., caducifolios, monoicos, con látex; indumento de pelos simples o con forma de T; corteza grisácea clara, áspera a casi lisa; ramas jóvenes pubescentes, luego glabras, robustas, con lenticelas prominentes. Hojas alternas, agrupadas hacia el extremo de las ramas; pecíolos tan largos como las láminas, con 2 glándulas apicales, glabrescentes; láminas variables, ovadas, enteras a 3-lobadas hacia el ápice, de (5-) 10-20 cm long. × (8-) 10-19 cm lat., primero puberulentas, luego la cara adaxial glabra, verde oscura, la abaxial, pubescente, verde grisácea, ápice agudo o acuminado, margen entero, base truncada o ligeramente cordada, nervios principales 3 (-7). Inflorescencias terminales en panículas de cimas achatadas en el ápice, con varias flores estaminadas y una flor pistilada terminal, aparecen antes que las hojas. Flores estaminadas con pedicelos de 2-3 cm long.; cáliz 2-lobado, espatáceo, ca. $10 \mathrm{~mm}$ long., castaño puberulento por fuera; pétalos 5 , obovados de 2-3 cm long. $\times 1-1,5 \mathrm{~cm}$ lat., blancos, con nervios rosados a purpúreos, unguiculados; estambres $8(-10)$, en 2 series, los internos con filamentos unidos por debajo de la mitad de su longitud, $c a$. 1,5 mm long., los externos de la mitad de su largo, libres. Flores pistiladas con cáliz y corola como en las estaminadas; ovario 3-5 (-8) locular, pubescente; óvulos 1 por lóculo; estilos 3-5(-8). Fruto drupa subglobosa, de 4-6 (-8) cm diám., apiculada; exocarpo crustáceo, liso, glabro, castaño rojizo. Semillas 3-4 (-8), ovoidecomprimidas, de 2-2,5 cm long. $\times 2-2,2 \mathrm{~cm}$ lat., algo verrucosas, con costillas dorsales y ventrales, castañas. $2 n=22$ (Huang et al., 1989).

Nombres vulgares. "Árbol del tung", "nuez de tung", "tung", "you tong" (China).

Distribución y hábitat. Especie del sur de China continental, Myanmar y norte de Vietnam, en general, de bosques abiertos, 200-1500 (-2000) m s.m., cultivada en las zonas cálidas del mundo (RadcliffeSmith, 1973, 1986; Li \& Gilbert, 2008; Welzen \& Chayamarit, 2012). Se introdujo en las primeras décadas del siglo XX en los Estados Unidos, Paraguay y la Argentina, con fines industriales (Jarvis, 2002; Brown \& Keeler, 2005).

Se encuentra naturalizada en los Estados Unidos (en el sudeste del país, California, Puerto Rico, Islas Vírgenes), Antillas, Paraguay, Georgia, India, Japón, Taiwán, Mozambique, Zimbabwe, Sudáfrica, Australia y Nueva Zelanda; a menudo deviene maleza o invasora (Wells et al. 1986; Liogier \& Martorell, 2000; Randall, 2012; Serviss et al., 2007; Hyde et al., 2012; Tropicos, 2013). En la Argentina, ha sido hallada naturalizada en Misiones, departamentos de Guaraní, Montecarlo, Libertador Gral. San Martín, Cainguás, San Ignacio, Leandro N. Alem, Candelaria y San Javier, en terrenos modificados, cercanías de plantaciones, capueras o bosques secundarios y sotobosques de monocultivos forestales.

Fenología. Florece en primavera, antes de la foliación; fructifica hasta el otoño. El polen es pesado y pegajoso. Las flores son polinizadas por coleópteros que permanecen, por lo común, en una misma planta, favoreciendo la autofecundación (Stockar, 1964).

Usos. El aceite de sus semillas tiene aplicaciones industriales. El residuo de la expresión de las semillas se utiliza como fertilizante: contiene más de un $25 \%$ 


\section{H. A. Keller et al. - Euphorbiaceae naturalizadas en la Argentina}
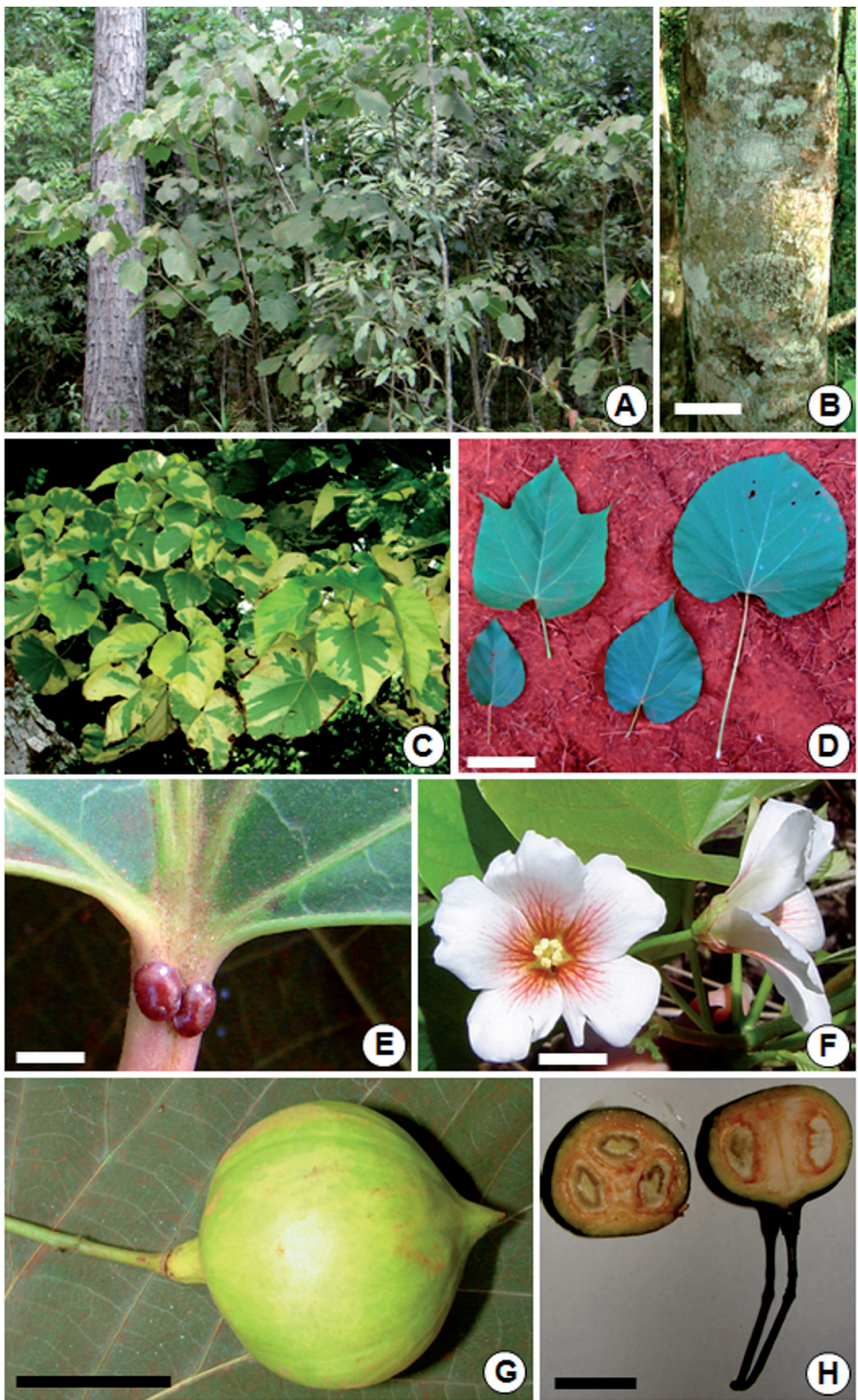

Fig. 1. Vernicia fordii. A: Regeneración espontánea bajo monocultivo forestal. B: Tronco. C: Ejemplar con hojas variegadas. D: Variación en la morfología foliar. E: Glándulas del pecíolo. F: Flores. G: Fruto. H: Frutos abiertos mostrando las semillas. A, D y E: Keller \& Paredes 10558. C: Keller \& Paredes 10625. F: Keller \& al. 4294. G y H: Keller \& Paredes 10577 (Escalas en cm: B: 10, D: 8, E: 0,4, F: 1,5, G y H: 4.). 
de proteínas; no obstante, no se usa para alimentar el ganado por su toxicidad. La corteza contiene taninos (Goldblatt, 1959; Stockar, 1964; Hanelt, 2001; Pastor, 2011). Se cultiva como ornamental; se reproduce por semillas y se multiplica por estacas (Dimitri, 1988). Presenta diversos cultivares (Li et al., 2008).

En China, el aceite de tung se ha usado tradicionalmente para lámparas, base de pinturas, tintas, impermeabilizantes para madera y papel, y en la construcción de la "Gran Muralla" (Blackmon, 1947; Goldblatt, 1959). En la medicina tradicional china, se aplica como antidermatósico, vulnerario y antiséptico (Duke \& Ayensu, 1985). Estudios fitoquímicos revelaron que el aceite tiene potencial efecto de activación del virus de Epstein-Barr (Ito et al., 1983). Las semillas contienen sustancias tóxicas (Okuda et al., 1975; Lin et al., 1996); sin embargo, se han usado como remedio drástico, emético y vermífugo (Welzen \& Chayamarit, 2012).

Importancia local. La historia de la actividad "tunguera" local denota un vínculo muy estrecho entre esta especie y los pobladores rurales e indígenas. En sus notas de campo inéditas, recogidas entre los guaraníes de Misiones, Raúl Martínez Crovetto registró la expresión híbrida "tũ 'a" (tũ: 'tung' y a: 'fruto') utilizada por los mbya guaraní para denominar al fruto del tung. En la actualidad, con sorprendente precisión taxonómica, los mbya utilizan la expresión "tũ rã" ('similar al tung') para denominar a Croton urucurana Baill., "sangre de drago", árbol nativo de la misma familia. Curiosamente, la nomenclatura botánica ha tomado el nombre tupí-guaraní para el epíteto específico: uruku-rã: 'similar al urucú' (Bixa orellana L.). C. urucurana se distingue fácilmente de $V$. fordii, entre otros caracteres, por sus hojas ovadas, anaranjadas al envejecer, inflorescencias racemosas, brácteas basales con una flor central pistilada y 2-3 estaminadas, las apicales sólo con flores estaminadas, estambres 16-20, y fruto piloso (Bacigalupo, 2005).

La introducción de $V$. fordii ha dado lugar, asimismo, a la conformación de topónimos locales. El paraje Tungoil, es una población rural concentrada alrededor de una antigua fábrica de aceite de tung, fundada en 1946 por algunos colonos con el nombre de "Santo Pipó Tungoill”, Departamento San Ignacio, municipio y colonia Santo Pipó (Stefañuk, 2009).

Los troncos y ramas se emplean como leña en secaderos de yerba mate y té, y los residuos resultantes de la extracción del aceite, se usan como abono en huertos y jardines. Se cultiva como ornamental, y se hallaron algunos ejemplares adventicios de hojas variegadas (Fig. 1C).

Naturalización. Los primeros ejemplares fueron introducidos en Misiones entre 1928 y 1930, por viveros particulares, como Pindapoy (Departamento San José) y el de Adolfo Schwelm, fundador de Eldorado. Hacia 1950, alcanzó un máximo de 54.820 ha cultivadas. Después de la crisis de la década de 1960, con la caída de los precios internacionales del aceite de tung (suplantado por aceites sintéticos), la actividad decreció hasta la actualidad. El Alto Paraná es la zona de cultivo más importante, le siguen San Ignacio y Oberá (Belastegui, 2006).

La naturalización se ve favorecida por su autopolinización y autofertilización (un solo árbol puede generar una población entera), y puede producir frutos a los 3 años de edad. Debido a la toxicidad de las semillas, la dispersión zoocora es limitada o ausente (Serviss \& al., 2007). En la provincia, la resiembra espontánea es sorprendente en torno a las antiguas áreas de cultivo, donde se observan numerosos renovales. Es una de las especies más frecuentes en capueras, bosques secundarios y sotobosques de monocultivos forestales (Fig. 1A).

Obs. Al mismo tiempo que $V$. fordii, Adolfo Schwelm introdujo en Misiones a $V$. montana Lour., pero su cultivo no prosperó (Stockar, 1964), y tampoco se hallaron ejemplares adventicios. A diferencia de $V$. fordii, son árboles hasta de $20 \mathrm{~m}$ alt., perennifolios, dioicos, con frutos ovoides a subglobosos, exocarpo rugoso y estriado. Originaria de China y el Sudeste asiático (Li \& Gilbert, 2008), se ha naturalizado en África y los Estados Unidos (Randall, 2012).

Material examinado. ARGENTINA. Prov Misiones. Dpto. Guaraní, ruta provincial 13, a unos $25 \mathrm{~km}$ de El Soberbio, 28-VIII-2007, fl, Keller et al. 4293 (CTES).- Dpto. Montecarlo, Colonia Guatambú, 2-I-2012, fr, Keller \& Paredes 10558 (CTES).- Dpto. Libertador Gral. San Martín, ruta nacional $12, \mathrm{ca}$. El Alcazar, $26^{\circ} 41^{\prime} 22,8^{\prime \prime} \mathrm{S}-54^{\circ}$ 49' 01,4” W, 02-I-2012, fr, Keller \& Paredes 10559 (CTES); acceso a aldea guaraní Teko'a Miri, 11-I2012, fr, Keller \& Paredes 10574 (CTES).- Dpto. Cainguás, ruta nacional 14, entre Campo Grande y Aristóbulo del Valle, 11-I-2012, fr, Keller \& Paredes 
10577 (CTES).- Dpto. San Ignacio, ruta provincial 7, cerca de la aldea guaraní Virgen María, 11-I-2012, fr, Keller \& Paredes 10575 (CTES); ruta provincial 6, $27^{\circ} 15^{\prime} 40,2^{\prime}$ ' S - 55 $15^{\circ}$ '17,4" W, 11-I-2012, fr, Keller \& Paredes 10597 (CTES).- Dpto. Leandro N. Alem, alrededores de la ciudad de Alem, 25-I-2012, fr, Keller \& Paredes 10614 (CTES).- Dpto. Candelaria, Bonpland, 25-I-2012, fr, Keller \& Paredes 10613 (CTES).- Dpto. San Javier, $27^{\circ} 51$ ' 12,8” S - 55 13' 44,7” W, 25-I-2012, fr, Keller \& Paredes 10625 (CTES).

2. Aleurites moluccanus (L.) Willd., Sp. Pl. 4: 590, 1805. Fig. 2, A-H.

\section{var. moluccanus}

Jatropha moluccana L., Sp. Pl. 2: 1006, 1753.

(Para una lista completa de sinónimos ver: Li \& Gilbert, 2008; Tropicos, 2013).

Iconografia: Li \& Gilbert, 2008: fig. 319, 1-4.

Árboles hasta de $20 \mathrm{~m}$ alt., perennifolios, monoicos, con látex; indumento de pelos estrellados; corteza grisácea, rugosa o casi lisa; ramas jóvenes con indumento denso, castaño grisáceo, luego subglabras. Hojas alternas, con pecíolos de 5-12 (-22) cm long., puberulentos, ápice con 2 glándulas; láminas ovadas a elíptico-lanceoladas, a veces orbicular-reniformes, enteras o hasta 5-lobadas, de (7-) 14- 20 (-30) cm long. $\times(4-)$ 7-17 (-25) cm lat., papiráceas, puberulentas a glabrescentes, agudas a acuminadas, borde entero o crenado-dentado, base anchamente cuneada $u$ obtusa, rara vez cordada; nervios principales 3-5 (-7). Inflorescencias terminales paniculadas, de 10$20 \mathrm{~cm}$ long. $\times 10-14 \mathrm{~cm}$ lat., brácteas inferiores subtendiendo grupos de flores estaminadas, cada eje mayor terminado en una flor pistilada solitaria. Flores estaminadas con pedicelos de (5-) 7-10 mm long.; cáliz irregularmente 2-3 (-4) lobado, lobos de 2-4 mm long., agudos, tomentosos en el envés; pétalos 5, oblongo-espatulados de 5-8 mm long. $\times$ ca. $2 \mathrm{~mm}$ lat., obtusos, glabros, blanco amarillentos; estambres 15-20, en 3-4 series; filamentos internos en columna pilosa, ca. $4 \mathrm{~mm}$ long.; los externos, ca. $1 \mathrm{~mm}$ long., puberulentos. Flores pistiladas con pedicelos de 2-4,5 mm long.; cáliz y corola como en las flores estaminadas, pero los pétalos $c a$. 1,5 mm lat.; ovario 2 locular, ca. $2 \mathrm{~mm}$ diám., densamente piloso; óvulos 1 por lóculo; estilos 2. Fruto drupa subglobosa, de 4-6 $\mathrm{cm}$ diám., levemente 2-lobada, no apiculada; exocarpo ligeramente carnoso, con 4 costillas longitudinales inferiores, pubescente, verde oliváceo a castaño. Semillas 1-2, ovoides, de 2,5-3 cm alt. $\times 2,7-3 \mathrm{~cm}$ lat., superficie tuberculada, margen lateral aplanado, castaña o grisácea con motas castañas. $2 n=22,44$ (Huang et al., 1989; Sarkar, 1989).

Nombres vulgares. "Árbol candil”, "lumbán”, "tung comestible", "shi li" (China), "nogueira-dolitoral", "nogueira-de-Iguape" (Brasil), "lauci" (Fiji), "kemiri”" (Borneo), "kukuil" (Hawai).

Distribución y hábitat. Especie nativa de la India, Sri Lanka, Tailandia, Vietnam, Camboya, China continental, Taiwán, Indonesia, Filipinas, Polinesia y Nueva Zelanda; crece en bordes de caminos, bosques mixtos y perturbados, entre 100-1000 m s.m.; se introdujo en las zonas cálidas del mundo (Radcliffe-Smith, 1986; Li \& Gilbert, 2008; Welzen \& Chayamarit, 2012).

Se encuentra naturalizada en los Estados Unidos (Florida, Hawai, Puerto Rico y las Islas Vírgenes), Antillas, Brasil, Paraguay, Zimbabwe, Mozambique, Madagascar, Swazilandia, Australia y diversas islas del Pacífico; a menudo deviene maleza o invasora (Liogier \& Martorell, 2000; Randall, 2012; Hyde et al., 2012; Tropicos, 2013). En la Argentina, ha sido hallada naturalizada en Misiones, departamentos Eldorado y San Ignacio; y Corrientes, Departamento Santo Tomé, en sitios alterados en torno a cultivos, parques y jardines.

Fenología. Florece desde la primavera hasta el otoño; fructifica en otoño. La polinización es entomófila.

Usos. El aceite de las semillas se utiliza para fabricar barnices, pinturas, jabones, lubricantes. Se ha estudiado su uso en el control de termitas (Nakayama \& Osbrink, 2010) y como antibacteriano (Locher et al., 1995). Las semillas contienen ácido hidrociánico y trazas de toxoalbúminas ( $\mathrm{Li} \&$ Gilbert, 2008), que deben eliminarse para obtener un aceite comestible. Las semillas se comen cocidas o asadas, en poca cantidad, para evitar sus efectos tóxicos (Facciola, 2001).

Se cultiva como ornamental, se reproduce por semillas y se multiplica por estacas (Dimitri, 1988). Flores, frutos, corteza y raíces se utilizan en medicina tradicional para combatir catarros, asma, debilidad, diarrea, disentería, cefalalgias, fiebres, gonorrea, 
Bol. Soc. Argent. Bot. 48 (3-4) 2013
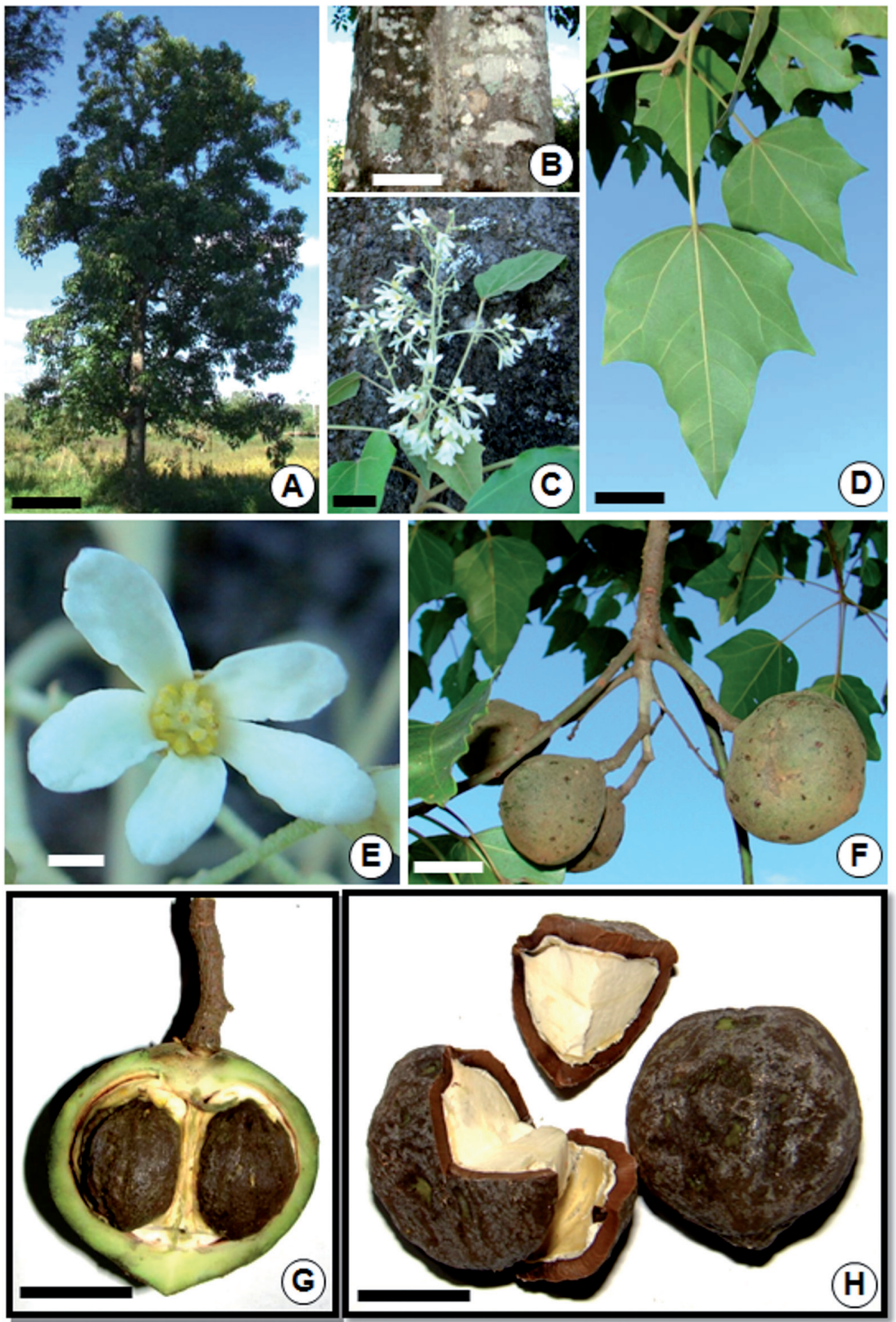

Fig. 2. Aleurites moluccanus. A: Ejemplar adulto en parque Schwelm, Eldorado. B: Tronco. C: Rama florida. D: Hojas. E: Flor. F: Frutos. G: Fruto abierto mostrandos las semillas. H: Semillas. A-l: Keller \& Paredes 10666 (Escalas en cm: B: 15; C y H: 2; D: 3; E: 0,1; F y G: 2,5). 


\section{H. A. Keller et al. - Euphorbiaceae naturalizadas en la Argentina}

heridas, úlceras, constipación, artritis y reumatismo; el aceite, en uso tópico, es antidermatósico y vulnerario (Weiner, 1970, 1971; Nagata, 1971; Uhe, 1974). La madera se utiliza para fabricar cajas de té y canoas. Las semillas se emplean como purgante e ictiotóxico; del endocarpo se obtiene un colorante negro. En Hawai, el endocarpo se emplea a modo de candela. El látex se ha usado como pegamento. El residuo de las semillas es usado como fertilizante (León, 1987; Hanelt, 2001).

Importancia local. Se ha cultivado por sus semillas y como ornamental; su relevancia es menor que la de V. fordii.

Naturalización. Los primeros ejemplares fueron introducidos en Misiones por los viveros particulares de Adolfo Schwelm y Pindapoy, entre 1928 y 1930, al igual que $V$. fordii. Se ha naturalizado de igual modo: se resiembra espontáneamente con facilidad en las zonas aledañas a su cultivo, parques y jardines; no obstante, su frecuencia y abundancia son menores.

Material examinado. ARGENTINA. Prov. Misiones. Dpto. Eldorado, Eldorado, parque Schwelm, 27-I-2012, fl, fr, Keller \& Paredes 10666 (CTES).Dpto. San Ignacio, paraje Teyú Cuaré, 29-III-2011, fl, fr, Keller \& Stampella 10051 (CTES).- Prov. Corrientes, Dpto. Santo Tomé, 28 30' $48^{\prime \prime}$ S - 56 03' 38” W, 19-II-2012, fl, fr, Keller 10794 (CTES).

\section{Clave de las especies}

1. Árboles caducifolios hasta de $10 \mathrm{~m}$ alt.; indumento de pelos simples o con forma de T. Estambres 8 $(-10)$, en 2 series. Ovario 3-5 (-8) locular; estilos 3-5 (-8). Fruto apiculado, exocarpo crustáceo, liso, glabro, castaño rojizo. Semillas 3-4 (-8), ligeramente verrucosas, castañas.

\section{Vernicia fordii}

1'. Árboles perennifolios hasta de $20 \mathrm{~m}$ alt.; indumento de pelos estrellados. Estambres 15-20, en 3-4 series. Ovario 2-locular, estilos 2. Fruto no apiculado, exocarpo ligeramente carnoso, con 4 costillas longitudinales inferiores, pubescente, verde oliváceo a castaño. Semillas $1-2$, tuberculadas, castañas o moteadas.

\section{Aleurites moluccanus}

\section{Discusión y Conclusiones}

Vernicia fordii y Aleurites moluccanus se consideran naturalizadas, dado que han logrado dispersarse por sus propios medios, establecerse e integrarse a la flora local, sin intervención humana por varias décadas. Algunas especies naturalizadas devienen invasoras, si su expansión compromete la funcionalidad y la biodiversidad de las comunidades naturales, por lo común, reemplazando a las especies autóctonas (Richardson et al., 2000; Pyšek \& al., 2004; Randall, 2012). Este no es el estado actual de estas especies en la Argentina, aunque se han registrado como invasoras en otros países. Su primer registro es entonces relevante, a fin de evaluar su potencial invasor en el futuro, sobre todo, considerando que la dinámica de las invasiones es un tema prioritario en diversos países (Pyšek \& Richardson, 2006; Rejmánek, 2000).

Los estudios etnobotánicos aportaron valiosa información sobre la introducción y naturalización de estas especies. Como se ha ensayado en estudios ecológicos en otros sitios (Lane, 1997), los relevamientos etnobotánicos suplementan los registros históricos y observacionales. La conjunción de los trabajos etnobotánicos y florísticos en el estudio del proceso de naturalización, constituye una aproximación novedosa, a la cual los autores han aportado previamente (Delucchi \& Keller, 2010; Keller et al., 2011; Hurrell et al., 2011, 2012).

\section{Agradecimientos}

A Daphne Colcombet por la información suministrada. A los pobladores locales por su desinteresada colaboración en la realización de este estudio. A los revisores anónimos por sus oportunas sugerencias.

\section{Bibliografía}

ALBUQUERQUE, U. P. \& R. F. LUCENA. 2004. Métodos e técnicas na pesquisa etnobotânica. Livro Rápido/NUPEEA, Recife.

BACIGALUPO, N. M. 2005. Euphorbiaceae. En: Burkart, A. \& M. N. Bacigalupo (eds.), Flora Ilustrada de Entre Ríos (Argentina). Col. Cient. Inst. Nac. Tecnol. Agropecu. 6 (4b): 101-195.

BELASTEGUI, H. M. 2006. Los colonos de Misiones. 
Ed. Universitaria, Posadas.

BLACKMON, G. H. 1947. Tung oil. A gift of China. Econ. Bot. 1: 161-175.

BROWN, K. \& W. KEELER. 2005. The History of Tung Oil. Wildland weeds (Winter): 2-6.

DELUCCHI, G. \& H. KELLER. 2010. La naturalización del "níspero", Eriobotrya japonica (Rosaceae, Maloideae), en la Argentina. Bonplandia 19: 71-77.

DIMITRI, M. J. 1988. Aleurites. En: Encicl. Argent. Agric. Jard. I (2): 241. Acme, Buenos Aires.

DUKE, J. \& E. AYENSU. 1985. Medicinal Plants of China. Reference, Algonac.

FACCIOLA, S. 2001. Cornucopia II. A source book of edible plants. 2da. impr. Kampong Publ., Vista.

GOLDBLATT, L. A. 1959. The tung industry. II. Processing and utilization. Econ. Bot. 13: 343-364.

GOVAERTS, R., D. G. FRODIN \& A. RADCLIFFESMITH. 2000. World Checklist and Bibliography of Euphorbiaceae and Pandaceae. Royal Bot. Garden, Kew.

HANELT, P. (ed.). 2001. Mansfeld's encyclopedia of agricultural and horticultural crops (except ornamentals) 3: 1216-1219. Springer, Berlin.

HUANG, S., Z. ZHAO, Z. CHEN, S. CHEN \& X. HUANG. 1989. Chromosome counts on one hundred species and infraspecific taxa. Acta Bot. Austro Sin. 5: 161-176.

HURRELL, J. A., G. DELUCCHI \& H. A. KELLER. 2011. Carya illinoinensis (Juglandaceae) adventicia en la Argentina. Bonplandia 20: 47-54.

HURRELl, J. A., G. DELUCCHI, H. A. KELlER, P.C. STAMPELLA \& E.L. GUERRERO. 2012. Bryophyllum (Crassulaceae): especies ornamentales naturalizadas en la Argentina. Bonplandia 21: 73-85.

HYDE, M. A., B. T. WURSTEN \& P. BALLINGS. 2012. Flora of Zimbabwe [online]. Disponible: http:// www.zimbabweflora.co.zw [Acceso: 15-III-2013].

IBODA. 2011. Instituto de Botánica Darwinion. Base de datos. Flora del Cono Sur [online]. Disponible: http// www2.darwin.edu.ar [Acceso: 18-III-2013].

ITO, Y., S. YANASE, H. TOKUDA, M. KISHISHITA, H. OHIGASHI, M. HIROTA \& K. KOSHIMIZU. 1983. Epstein-Barr virus activation by tung oil, extracts of Aleurites fordii and its diterpene ester 12-O-hexadecanoyl-16-hydroxyphorbol-13-acetate. Cancer Lett. 10: 87-95.

JARVIS, A. J. 2002. Paraguayan Tung (Aleurites fordii Hemsl.). An important small farmer. Technological University, Michigan.

KELLER, H. A., G. DELUCCHI \& H. ROMERO. 2011. Camellia sinensis (Theaceae) en la Argentina: naturalización y usos locales. Bol. Soc. Argent. Bot. 46: $145-150$

LANE, R. 1997. Oral histories and scientific knowledge in understanding environmental change: a case study in the Tumut Region, New South Wales, Australia. Geographical Res. 35: 195-205.

LEÓN, J. 1987. Botánica de los cultivos tropicales. IICA, San José de Costa Rica.

LI, B. \& M. G. GILBERT. 2008. Aleurites, Vernicia. In: Wu, Z. Y., P. H. Raven \& D. Y. Hong (eds.), Flora of China 11: 265-267. Science Press, Beijing-Missouri Botanical Garden Press, St. Louis.

LI, P., X. ZHANG, Y. CHEN, G. LU, G. ZHOU \& Y. WANG. 2008. Genetic diversity and germplasm resource research on tung tree (Vernicia fordii) cultivars, investigated by inter-simple sequence repeats. African J. Biotechnol. 7: 1054-1059.

LIN, T., C. HSU, L. SHIU \& J. DENG. 1996. Two outbreaks of acute Tung Nut (Aleurites fordii) poisoning. J. Toxicol. Clin. Toxicol. 34: 87-92.

LIOGIER, H. A. \& L. F. MARTORELL. 2000. Flora of Puerto Rico and adjacent islands. A systematic synopsis. 2nd ed. Edit. Universidad de Puerto Rico, San Juan.

LOCHER, C, M.BURCH,H.MOWER, J.BERESTECKY, H. DAVIS, B. VAN POEL, A. LASURE, D. VANDEN BERGHE \& A. VLIETINCK. 1995. Antimicrobial activity and anti-complement activity of extracts obtained from selected Hawaiian medicinal plants. J. Ethnopharmacol. 49: 23-32.

NAGATA, K. 1971. Hawaiian medicinal plants. Econ. Bot. 25: 245-254.

NAKAYAMA, F. S. \& W. L. OSBRINK. 2010. Evaluation of kukui oil (Aleurites moluccana) for controlling termites. Industr. Crops Prod. 31: 312-315.

OKUDA, T., T. YOSHIDA, S. LOIKE \& N. TOH. 1975. New diterpene esters from Aleurites fordii fruits. Phytochemistry 14: 509-515.

PASTOR, S. 2011. Determining biological roles of four unique Vernicia fordii acyl-CoA binding proteins. Theses and Dissertations, Paper 1337: 1-123. University of New Orleans, New Orleans.

PYŠEK, P. \& D. M. RICHARDSON. 2006. The biogeography of naturalization in alien plants. $J$. Biogeogr. 12: 2040-2050.

PYŠEK, P., D. M. RICHARDSON, M. REJMÁNEK, G. WEBSTER, M. WILLIAMSON \& J. KIRSCHNER. 2004. Alien plants in checklists and floras: towards better communication between taxonomists and ecologists. Taxon 53: 131-143.

RADCLIFFE-SMITH, A. 1973. Typification of Vernicia fordii (Euphorbiaceae). Kew Bull. 28: 296.

RADCLIFFE-SMITH, A. 1986. Euphorbiaceae. En: E. Nasir \& S. I. Ali (eds.), Flora of Pakistan 172: 1-164. Univ. of Karachi, Karachi.

RADCLIFFE-SMITH, A. 2001. Genera euphorbiacearum. Royal Bot. Garden, Kew.

RANDALL, R. 2012. A Global Compendium of Weeds. 2nd ed. Dep. Agr. Food, Perth. 


\section{H. A. Keller et al. - Euphorbiaceae naturalizadas en la Argentina}

REJMÁNEK, M. 2000. Invasive plants: approaches and predictions. Austral Ecol. 25: 497-506.

RICHARDSON, D. M., P. PYŠEK, M. REJMÁNEK, M. G. BARBOUR, F. DANE PANETTA \& C. J. WEST. 2000. Naturalization and invasion of alien plants: concepts and definitions. Diversity Distrib. 6: 93-107.

SARKAR, A. K. 1989. Cytological assessment of the family Euphorbiaceae. III. Subtribe Jatropheae. Proc. Indian Sci. Congr. Assoc. 76 (3,VI): 183.

SECCO, R. S., I. CORDEIRO, L. SENNA, M. SALES, L. RIBES, D. MEDEIROS, B. HAIAD, A. S. OLIVEIRA, M. B. CARUZO, D. CARNEIROTORRES \& N. C. BIGIO. 2012. An overview of recent taxonomic studies on Euphorbiaceae sensu lato in Brazil. Rodriguésia 63: 227-242.

SERVISS, B., N. FREEMAN, J. HERNANDEZ, A. LEIBLE \& C. TALLEY. 2007. Tungoil Tree (Alellrites fordii Hemsl.) (Euphorbiaceae). New to the Arkansas Flora. J. Arkansas Acad. Sci. 61: 128130.

STEFAÑUK, M. A. 2009. Diccionario geográfico toponímico de Misiones. Contratiempo Ed., Buenos Aires.

STOCKAR, A. 1964. Tung. En: Encicl. Argent. Agric. Jard. II: 697-706. Acme, Buenos Aires.

TROPICOS. 2013. Tropicos.org. Missouri Botanical Garden. [online]. Disponible: http//www.tropicos. org [Acceso: 18-III-2013].

STUPPY, W., P. C. VAN WELZEN, P. KLINRATANA \& M. POSA. 1999. Revision of the genera Aleurites, Reutealis and Vernicia (Euphorbiaceae). Blumea 44: 73-98.

THAKUR, H. A. \& D. A. PATIL. 2011. Taxonomic and phylogenetic assessment of the Euphorbiaceae: a review. J. Exp. Sci. 2: 37-46.
TOKUOKA, T. 2007. Molecular phylogenetic analysis of Euphorbiaceae sensu stricto based on plastid and nuclear DNA sequences and ovule and seed character evolution. J. Plant Res. 120: 511-522.

UHE, G. 1974. Medicinal plants of Samoa. Econ. Bot. 28: 1-30.

WEINER, M. 1970. Notes on some medicinal plants of Fiji. Econ. Bot. 24: 279-282.

WEINER, M. 1971. Ethnomedicine in Tonga. Econ. Bot. 25: 423-450.

WELLS, M. J., V. BALSINHAS, H. JOFFE, V. M. ENGELBRECHT, G. HARDING \& C. STIRTON. 1986. A catalogue of problem plants in Southern Africa, incorporating the National Weed List of South Africa. Mem. Bot. Surv. S. Africa 53: 1-663.

WELZEN, P. C. VAN \& W. STUPPY. 1999. Phylogenetic considerations of Euphorbiaceae tribe Aleuritideae. Ann. Missouri Bot. Gard. 86: 894-903.

WELZEN, P. C. VAN \& K. CHAYAMARIT. 2012. Flora of Thailand: Euphorbiaceae [online]. Disponible: http://www.nationalherbarium.nl/thaieuph [Acceso: 15-III-2012].

WURDACK, K. J., P. HOFFMANN \& M. W. CHASE. 2005. Molecular phylogenetic analysis of uniovulate Euphorbiaceae (Euphorbiaceae sensu stricto) using plastid RBCL and TRNL-F DNA sequences. Amer. J. Bot. 92: 1397-1420.

Recibido el 10 de agosto de 2012, aceptado el 3 de abril de 2013. 
